\title{
Themenvielfalt und Themenspezialisierung
}

\section{Dennis Edler ${ }^{1}$}

Accepted: 5 May 2021 / Published online: 31 May 2021

(c) The Author(s) 2021

Liebe Leserinnen und Leser,

wie die englische Bezeichnung dieser Zeitschrift, „KN - Journal of Cartography and Geographic Information“, hervorhebt, richtet sie sich zwar primär, aber nicht ausschließlich an kartographischen Fachfragen aus. Das fachlich umfangreiche Feld der Geoinformationswissenschaften bietet viele themen- und methodenverwandte Fragestellungen, die diese Fachzeitschrift bereichern. Dabei bildet die Verarbeitung digitaler Geodaten das entscheidende und zeitgemäße Bindeglied, das raumbezogene Disziplinen vernetzt und das Themenspektrum für moderne Fachzeitschriften der Kartographie und Geomatik erweitert.

Dass die KN eine Zeitschrift der Themenvielfalt aus der Kartographie und den Geoinformationswissenschaften darstellt, verdeutlichen die zurückliegenden Jahre seit dem Verlagswechsel zu Springer. Varia-Hefte bilden den eigentlichen Kern bei der Herausgabe neuester wissenschaftlicher Erkenntnisse in den KN, was die gewünschte Themenvielfalt unterstützt und auch sicherlich - je nach Thema der einzelnen Beiträge - für einen gewissen Spannungs- und Überraschungsmoment bei der Leserschaft sorgt.

Ein genauerer Blick auf andere etablierte Fachzeitschriften der Kartographie und Geoinformationswissenschaften verdeutlicht jedoch auch, dass - neben der Unterstützung von Themenvielfalt durch Varia-Hefte - auch die Themenspezialisierung durch regelmäßige Sonderhefte (,Special Issues“) zunimmt. Dadurch werden aktuelle Anwendungs- und Methodenfelder gestärkt, und die veröffentlichten Ausgaben erfahren durch gezieltere Verbreitung im Web (bspw. durch angepasste Webseitenauftritte der Zeitschriften und Posts in Sozialen Medien) eine höhere Sichtbarkeit, was wiederum ein entscheidendes Kriterium für international aktive Forschungsteams sein kann, sich einem Themenheft anzuschließen. Ein jüngstes Beispiel ist

\footnotetext{
Dennis Edler

Dennis.Edler@ruhr-uni-bochum.de

1 Bochum, Germany
}

das im Frühjahr 2021 veröffentlichte Themenheft „Mapping the Pandemic" in der kanadischen Fachzeitschrift „Cartographica“ (Jg. 56, H. 1).

Die Themenspezialisierung durch Themenhefte ist in Bezug auf die KN kein Novum. Bereits in der Vergangenheit gab es, auch unter Einbindung von Gastherausgeberinnen und -herausgebern, entsprechende Ausgaben, wie bspw. Hefte zur Audiovisuellen Kartographie (Jg. 69, H. 1) und mit Bezug zur Mobilität im urbanen Raum (Jg. 70, H. 1). Um in Zukunft die Themenvielfalt und -sichtbarkeit des wissenschaftlichen Outputs sowie auch des Info- und Praxisteils der KN noch regelmäßiger durch Spezialisierungen mit Themenheften zu konzentrieren, sind Vorschläge aus der Community jederzeit herzlich willkommen! Für Heft 4 des laufenden Jahrgangs ist, betreut durch die Leiter der gemeinsamen DGfK-/DGPF-Kommission zu VR und AR, ein Themenheft „Virtual and Augmented Reality in Spatial Visualization“ (mit Beitragseinreichungen bis zum 01.08.) vorgesehen.

Die Fachartikel des aktuellen Varia-Heftes deuten auf die vielseitigen Darstellungs- und Einsatzmöglichkeiten von Geodaten in der Forschung: Mit einer empirischen Erhebung verdeutlicht Jochen Schiewe (Hamburg) u.a., dass Equal Area Unit Maps (EAUMs) die Erkennung lokaler Extremwerte signifikant verbessern. Ulrich Schumacher (Dresden) adressiert die zunehmende Urbanisierung und stellt einen GIS-basierten Ansatz vor, wie aus Daten des Urban Atlas eine europaweite Siedlungsmaske abgeleitet werden könnte. Das Team, bestehend aus Christian Röger, Maja Kalinic und Jukka M. Krisp (Augsburg), kombinieren Daten aus Verkehrszählungen (Inductive Loop Detector Data, ILD) mit Floating Car Data (FCD) und analysieren fallbeispielbezogen die Aussagekraft dieses methodischen Ansatzes. Nnamdi Ifeanyi Maduekwe (Ile-Ife) zeigt in einer GIS-basierten Methodik Extraktionspotenziale von digitalen Geodaten aus topographische Karten Nigerias. 
Sophie Grunenberg und Markus Hilpert (Augsburg) stellen ein Projekt zur historischen Kulturlandschaft Augsburgs vor, das Ergebnisse aus verschiedenen Datenressourcen bzw. - erhebungen zusammenführt.

Viel Freude beim Lesen und herzliche Grüße!

\section{Dennis Edler}

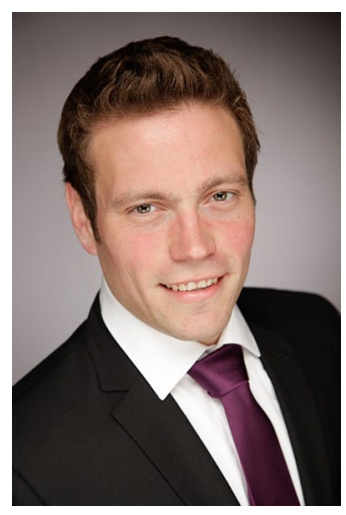

Funding Open Access funding enabled and organized by Projekt DEAL.

Open Access This article is licensed under a Creative Commons Attribution 4.0 International License, which permits use, sharing, adaptation, distribution and reproduction in any medium or format, as long as you give appropriate credit to the original author(s) and the source, provide a link to the Creative Commons licence, and indicate if changes were made. The images or other third party material in this article are included in the article's Creative Commons licence, unless indicated otherwise in a credit line to the material. If material is not included in the article's Creative Commons licence and your intended use is not permitted by statutory regulation or exceeds the permitted use, you will need to obtain permission directly from the copyright holder. To view a copy of this licence, visit http://creativecommons.org/licenses/by/4.0/. 